\title{
REVISIÓN \\ Ecología de invasiones marinas en Chile continental: ¿Qué sabemos y que nos falta por saber?
}

Ecology of marine invasions in continental Chile: What do we know and we need to know?

\author{
Cristóbal Villaseñor-Parada ${ }^{1,2,3,4}$, Aníbal Pauchard ${ }^{2,3}$ y Erasmo C. Macaya ${ }^{4,5,6}$ \\ 'Departamento de Botánica, Facultad de Ciencias Naturales y Oceanográficas, Universidad de Concepción, Casilla 160-C, Concepción, \\ Chile.cvillasenor@udec.cl \\ ${ }^{2}$ Laboratorio de Invasiones Biológicas (LIB), Facultad de Ciencias Forestales, Universidad de Concepción, Casilla 160-C, Concepción, \\ Chile \\ ${ }^{3}$ Instituto de Ecología y Biodiversidad (IEB), Facultad de Ciencias, Universidad de Chile, Las Palmeras 3425, Nuñoa, Santiago, Chile \\ ${ }^{4}$ Laboratorio de Estudios Algales (ALGALAB), Departamento de Oceanografía, Facultad de Ciencias Naturales y Oceanográficas, \\ Universidad de Concepción, Casilla 160-C, Concepción, Chile \\ ${ }^{5}$ Millennium Nucleus Ecology and Sustainable Management of Oceanic Island (ESMOI), Coquimbo, Chile \\ ${ }^{6}$ Centro FONDAP de Investigación en Dinámica de Ecosistemas Marinos de Altas Latitudes (IDEAL), Chile
}

\begin{abstract}
The Chilean coast has fewer reports of introduced species for other shores worldwide, which could correspond to a natural phenomenon generated by oceanographic conditions, or an artifact caused by lack of available information. We analyzed 71 papers indexed in Web of Science related with ecology of marine invasions in the Chilean coast, published between 1998-2014, and we determined the current state of the discipline in Chile, and identified the trends (e.g., temporal, spatial, taxonomic) of the investigation. Most of the papers studied species naturalization stage, and very few deal with invasive species. The number of papers per year increases linearly with time, suggesting a growing interest of the scientific community in the study of the ecology of marine invasions. The amount of available information (i.e., number of publications) is not homogeneous among administrative regions, and most papers have examined specific sectors within each region (usually near the regional capital). Regions with high maritime traffic (e.g., Valparaíso, Magallanes) have been scarcely studied. With the exception of Coquimbo and Los Lagos, researches by region have been performed on a few taxa. Hardly any studies cover large spatial scales, although several of them do perform monitoring programs on longer time scales (e.g., monthly, yearly). Our results suggest that the number of exotic species in Chile may be underestimated. Field observations, periodic updating of catalogs and use of molecular tools are proposed as measures to achieve a greater understanding of the issue at national level.
\end{abstract}

Key words: Conservation, naturalized species, invasive species, spatial scale, temporal scale

Resumen.- La costa chilena presenta menos reportes de especies introducidas respecto a otras costas a nivel mundial, lo que podría corresponder a un fenómeno natural generado por condiciones oceanográficas, o bien a un artefacto producido por falta de información disponible. Mediante el análisis de 71 trabajos indexados en Web of Science, relacionados con ecología de invasiones marinas en la costa chilena, publicados entre 1998-2014, determinamos el estado actual de esta disciplina en Chile, e identificamos las tendencias de las investigaciones realizadas. La mayoría de las publicaciones se relacionan con especies en etapa de naturalización, y pocas con especies invasoras. El número de publicaciones por año incrementa linealmente, sugiriendo un creciente interés de la comunidad científica respecto al tema. La cantidad de información disponible (i.e., número de publicaciones) no es homogénea entre las regiones administrativas, y la mayoría de los trabajos son realizados sectores puntuales dentro de cada región (usualmente cercanos a la capital regional). Regiones con alto tráfico marítimo (e.g., Valparaíso, Magallanes) han sido muy poco estudiadas. A excepción de la región de Coquimbo y Los Lagos, los trabajos por región apuntan a unos pocos taxa. Muy pocos trabajos abarcan grandes escalas espaciales, aunque varios de ellos realizan experimentos a escalas de tiempo mayores (e.g., mensual, anual). Nuestros resultados permiten sugerir que el número de especies reportadas como introducidas en Chile podría estar subestimado. Observaciones en terreno, actualización periódica de catastros y utilización de herramientas moleculares, son propuestos como medidas para una mayor comprensión del tema a nivel nacional.

Palabras clave: Conservación, especie naturalizada, especie invasora, escala espacial, escala temporal

\section{INTRODUCCIÓN}

Durante los últimos años se ha observado a nivel mundial un creciente aumento en el número de investigaciones relacionadas con ecología de invasiones a través del tiempo (Puth \& Post 2005, Lockwood et al. 2007), aunque el esfuerzo de investigación está fuertemente sesgado hacia Norteamérica y Europa (Pyšek et al. 2008). En sistemas marinos, la investigación en ecología de invasiones también ha aumentado temporalmente (Puth \& Post 2005, Davidson \& Simkanin 
2012), sin embargo, han sido bastante menos estudiados en comparación con sistemas terrestres y dulceacuícolas (Puth \& Post 2005).

En la comunidad científica de Chile, el interés en temas relacionados con ecología de invasiones también se ha incrementado (Quiroz et al. 2009), pero al igual como ocurre a nivel mundial, dicho aumento se ha dado principalmente en sistemas terrestres, mientras que sistemas marinos han sido menos investigados, y sólo recientemente abordados (Castilla et al. 2005, Castilla \& Neill 2009). A pesar de ello, la introducción de especies es considerada como una clara amenaza a la conservación de ecosistemas acuáticos chilenos (Gajardo \& Laikre 2003, Camus 2005).

Según Castilla \& Neill (2009), 51 especies en la costa de Chile continental son introducidas o al menos criptogénicas (i.e., especies de las que no puede demostrarse si son nativas o exóticas, sensu Carlton 1996), un número mucho menor al reportado para otras costas como Nueva Zelanda $(n=109$; Cranfield et al. 1998), Estados Unidos ( $\mathrm{n}=298$; Ruiz et al. 2000), Francia ( $n=104$; Goulletquer et al. 2002), Italia ( $n=$ 110; Ambrogi 2002) y Australia ( $\mathrm{n}=99$; Hewitt et al. 2004). Castilla \& Neill (2009) atribuyen este bajo número de especies introducidas a una resistencia natural de la costa chilena basado en sus características oceanográficas (e.g., sistema de corrientes frías a lo largo de toda la costa; zonas de mínimo oxígeno en el norte; baja cantidad de golfos, bahías y estuarios al norte de Chiloé; baja temperatura y salinidad en la zona de canales del sur de Chile) y una resistencia biótica (i.e., resistencia a la invasión provocada por la comunidad residente mediante interacciones negativas; Kimbro et al. 2013) generada por depredadores bentónicos.

Por otra parte, el número de reportes de especies introducidas en un país también puede depender de diferentes factores metodológicos y socio-culturales, como la dificultad de acceso al muestreo de diversos hábitats, la disponibilidad de registros históricos, el esfuerzo en investigación y el nivel de desarrollo del país (Cartlon 2009, McGeoch et al. 2010). Países en desarrollo como Chile, tienen menor cantidad de publicaciones relacionadas con invasiones que países desarrollados (Nuñez \& Pauchard 2010), y la cantidad de información disponible (e.g., número de publicaciones) se relaciona positivamente con el número de especies reportadas como introducidas (McGeoch et al. 2010). Por esta razón, conocer la cantidad y tipo de información que se ha generado en Chile respecto a ecología de invasiones marinas podría ayudar a clarificar si el bajo número de especies introducidas reportadas para Chile es producto de una resistencia natural de la costa chilena ante la invasión (e.g., características oceanográficas, resistencia biótica), o bien a un artefacto (i.e., factor que perturba la correcta interpretación del resultado) producto del menor esfuerzo en investigación y/o una desigual cantidad de información disponible a lo largo de la costa (i.e., sectores de la costa chilena menos estudiados que otros).

A partir de una búsqueda de la literatura relacionada con ecología de invasiones marinas en Chile, los objetivos de este trabajo fueron: a) conocer el estado actual respecto al tema, identificando los principales temáticas que se estudian en Chile, y b) analizar las tendencias temporales (número de publicaciones a través del tiempo), espaciales (número de publicaciones por región administrativa) y taxonómicas (principales taxa estudiados) de los trabajos vinculados a ecología de invasiones marinas, así como las escalas (espaciales y temporales) abarcadas en dichos estudios.

\section{Materiales Y MÉTODOS}

\section{A) Criterios de inclusión y exclusión de artículos}

Como una aproximación de la cantidad de información disponible para Chile, se utilizó el conjunto de publicaciones relacionadas directamente con ecología de invasiones marinas (e.g., reportes de nuevas especies, impactos ecológicos y económicos generados, distribución en la costa chilena, modelos), las cuales fueron obtenidas a partir de una exhaustiva búsqueda en ISI Web of Knowledge entre 1998 y 2014 (Web of Science $\left.{ }^{\circledR}\right)$. No se consideraron trabajos anteriores a 1998 pues en ese año se comenzó a abordar el tema de invasiones marinas en Chile por entidades como la Comisión Permanente del Pacífico Sur (e.g., Reunión de expertos para analizar los efectos ecológicos de la introducción de especies exóticas en el Pacífico SE, Báez et al. 1998 ). Además los trabajos con antelación a dicho año son bastante escasos (Castilla et al. 2005). En esta búsqueda se incluyeron artículos, revisiones y comentarios, publicados en revistas WOS nacionales y extranjeras, cuyos resultados estuvieran directamente relacionados con sistemas marinos de Chile continental. Fueron excluidos de este análisis trabajos relacionados netamente con la fisiología y obtención de productos naturales a partir de especies introducidas, así como también aquellos destinados a incrementar el rendimiento y mejorar las condiciones de cultivo en especies introducidas con fines comerciales como la ostra

${ }^{1}$ Báez PR, M Meléndez, S Ramírez, A Letelier, M Brown, C Campos, C Alday, C Jelvez \& A Alvial. 1998. Efectos ecológicos de la introducción de especies exóticas en el medio marino y costero chileno. En: Reunión de expertos para analizar los efectos ecológicos de la introducción de especies exóticas en el Pacífico sudeste, Viña del Mar, Chile, Comisión Permanente del Pacífico Sur, pp. 1-83. 
del Pacífico Crassostrea gigas (Thunberg, 1793); los abalones Haliotis discus hannai (Ino, 1953) y Haliotis rufescens (Swainson, 1822); o las especies de salmónidos Oncorhynchus mykiss (Walbaum, 1792), Oncorhynchus kisutch (Walbaum, 1792), Oncorhynchus tshawytscha (Walbaum, 1792) y Salmo salar (Linnaeus, 1758). No fueron excluidos de este análisis trabajos relacionados con estas especies que incluyeran aspectos relacionados con la conservación, o efectos sobre componentes bióticos del sistema (e.g., introducción de especies no objetivo, efectos sobre su fauna asociada, interacciones bióticas con especies nativas). En el caso de especies anádromas, como las especies de salmones introducidos en el sur de Chile, sólo se incorporaron aquellos trabajos en los cuales la totalidad, o al menos parte de los experimentos $\mathrm{y} / \mathrm{u}$ observaciones, fueron realizados en sistemas marinos, mientras que artículos que hacían referencia exclusivamente a sistemas dulceacuícolas no fueron incluidos. Trabajos relacionados con mamíferos introducidos asociados a sistemas marinos como el visón en el extremo sur de Chile, tampoco fueron incluidos.

\section{B) Clasificación de artículos y análisis de los DATOS}

Los trabajos (a excepción de aquellos en formato de revisión y/o comentarios) fueron clasificados según la temática abordada, la especie estudiada, la etapa en la que se encuentra en el proceso de invasión, la región administrativa en la que se realizaron los experimentos u observaciones, y la duración de los experimentos y/o muestreos. Un mismo trabajo podía abarcar más de una temática, especie exótica o región administrativa.

Los trabajos analizados fueron clasificados en 10 categorías según la temática abordada: a) primeros reportes, b) trabajos genéticos, c) depredación, d) facilitación, e) competencia, f) biología reproductiva, g) estrategias de dispersión y persistencia, h) efectos socio-económicos, i) modelos yj) distribución en la costa chilena.

Para determinar la etapa del proceso de invasión en la que se encuentra la especie estudiada, se siguió la clasificación propuesta por Blackburn et al. (2011), en donde se considera como especies exóticas a aquellas cuya presencia en una región biogeográfica se debe a la introducción intencional o accidental como consecuencia de la actividad humana, especies naturalizadas a aquellas especies exóticas que luego de haber sido introducidas se reproducen constantemente y mantienen poblaciones estables sin la intervención directa de los seres humanos, y especies invasoras a aquellas especies naturalizadas que tras su establecimiento se propagan desde su punto de introducción ocupando hábitats naturales, y que en algunos casos pueden dominar los hábitats invadidos. En este trabajo se considera especies exclusivamente exóticas a aquellas introducidas con fines de cultivo pero que no forman poblaciones naturales como la ostra Crassostrea gigas y los abalones Haliotis spp. En cuanto a las especies de salmones, en este trabajo las consideramos como naturalizadas, y no como invasoras, ya que la expansión y ocupación de hábitats naturales se da principalmente en cuencas de ríos y lagos.

La relación entre el número de publicaciones y el año de publicación fue evaluada mediante regresión lineal.

Los trabajos realizados en terreno fueron clasificados según las regiones políticas abarcadas en los experimentos $\mathrm{u}$ observaciones (en estudios cuyo objetivo era determinar la distribución de la especie exótica en la costa chilena, se consideraron todas aquellas regiones muestreadas aun cuando la especie exótica no se encontrara en dicha región). En trabajos que fueron realizados en laboratorio se consideró la región desde la cual fueron obtenidas las muestras. Trabajos que consistían en modelos (e.g., de nicho, socio-económicos) fueron incluidos sólo cuando se tomaron datos en terreno para parámetros puntuales.

\section{RESULTADOS Y DISCUSIÓN}

Los primeros trabajos que sintetizaron la información existente, y entregaron listados de especies marinas exóticas en Chile son relativamente recientes (e.g., Castilla et al. 2005, Castilla \& Neill 2009), y producto de la sinergia entre taxónomos y ecólogos. Estos trabajos (y sus correspondientes listados) constituyen la base para trabajos subsiguientes relacionados con invasiones en sistemas marinos chilenos.

En el catastro de Castilla et al. (2005) se identifican 47 especies exóticas y criptogénicas (15 de ellas deliberadamente introducidas para acuicultura), mientras que en el catastro de Castilla \& Neill (2009) el número se eleva a 51. Desde entonces, algunas revisiones realizadas para taxa particulares han efectuado nuevos reportes de especies exóticas no incluidas en los catastros anteriores (e.g., 6 especies de poliquetos perforadores, Moreno et al. 2006; 2 especies de ascidias, Turon et al. 2016; 8 especies de macroalgas marinas, Villaseñor-Parada $e t a l .{ }^{2}$ ). Por otra parte, algunos trabajos han clarificado el estatus para especies que se creían exóticas, pero en realidad correspondían a especies nativas (e.g., el poliqueto Polydora bioccipitalis (Blake \& Woodwick, 1972), Riascos et al. 2009; 6 especies de macroalgas, Villaseñor-Parada et $\left.a l^{2}\right)$. La revisión más reciente referente a especies introducidas

${ }^{2}$ Villaseñor-Parada C, A Pauchard, ME Ramírez \& EC Macaya. Macroalgas exóticas en la costa de Chile continental: patrones espaciales, temporales, taxonómicos y ecológicos. Latin American Journal of Aquatic Research [aceptado con cambios mayores] 
en Chile, ha identificado para sistemas marinos y dulceacuícolas un total de 21 especies de macro y microalgas, y 79 especies de moluscos, poliquetos y peces, de origen exótico (LIB $2016)^{3}$.
En nuestra búsqueda bibliográfica, se seleccionaron un total de 71 publicaciones WOS (ex ISI) (ver Suplemento) relacionadas directamente con ecología de invasiones en sistemas marinos de la costa de Chile continental publicados entre 1998 y 2014, en las cuales sólo se estudiaron 27 de estas especies (Tabla 1).

Tabla 1.- Número de trabajos por cada una de las especies exóticas en la costa chilena que han sido objetos de estudio en los artículos analizados, con información respecto a su estatus, las regiones administrativas donde se han realizado experimentoso búsquedas para cada una de ellas (simbología descrita en leyenda de la Fig. 3) y las temáticas abordadas en dichos trabajos (categorías son simbolizadas con letras descritas en Materiales y métodos) / Number of works for each of exotic species in the Chilean coast that have been subject of study in the analyzed papers, with information about their status, administrative regions where experiments or searches have been performed (symbology described in legend of Fig. 3 ) and the themes addressed in those work (categories are symbolized with letters described in Materials and methods)

\begin{tabular}{|c|c|c|c|c|c|}
\hline Phylum / Clase & Especie & Estatus & $\begin{array}{l}\mathrm{N}^{\circ} \mathrm{de} \\
\text { trabajos }\end{array}$ & $\begin{array}{c}\text { Regiones } \\
\text { administrativas }^{1}\end{array}$ & $\begin{array}{l}\text { Temáticas } \\
\text { abordadas }\end{array}$ \\
\hline Chlorophyta & Codium fragile subsp. fragile & Invasora & 10 & entre la II y la XII & $a, b, d, f, g, h, i, j$ \\
\hline Phaeophyceae & Scytosiphon tenellus & Naturalizada & 1 & III & $a, b$ \\
\hline \multirow[t]{4}{*}{ Rhodophyta } & Neosiphonia harveyi & Naturalizada & 1 & III & d \\
\hline & Polysiphonia morrowii & Naturalizada & 1 & XII & $a, b$ \\
\hline & Mastocarpus latissimus & Invasora $^{2}$ & 3 & entre la V y la XI & $g, j$ \\
\hline & Glaphyrosiphon chilensis $^{3}$ & Naturalizada & 1 & $\mathrm{~V}$ & $\mathrm{a}$ \\
\hline \multirow[t]{4}{*}{ Ascidacea } & Pyura praeputialis & Naturalizada & 18 & II & a,b,c,d,e,f,g,h,i \\
\hline & Ciona robusta & Invasora $^{4}$ & 8 & II, IV & $\mathrm{a}, \mathrm{c}, \mathrm{e}, \mathrm{f}, \mathrm{g}, \mathrm{h}, \mathrm{i}$ \\
\hline & Asterocarpa humilis & Naturalizada & 2 & II & $\mathrm{a}$ \\
\hline & Molgula ficus & Naturalizada & 2 & II & $\mathrm{a}$ \\
\hline \multirow{2}{*}{ Cnidaria } & Anemonia alicemartinae & Invasora & 3 & entre la XV y la VIII & $a, f, g, j$ \\
\hline & Aurelia aurita & Naturalizada & 1 & $\mathrm{XI}$ & $\mathrm{a}$ \\
\hline \multirow[t]{2}{*}{ Bivalvia } & Mytilus galloprovincialis & Naturalizada & 10 & entre la VIII y la XII & $a, b, f, j$ \\
\hline & Crassostrea gigas & Exótica & 3 & $\mathrm{X}$ & $\mathrm{i}, \mathrm{j}$ \\
\hline \multirow[t]{2}{*}{ Gastropoda } & Haliotis rufescens & Exótica & 8 & III, IV, V, X & $\mathrm{a}, \mathrm{d}$ \\
\hline & Haliotis discus hannai & Exótica & 8 & III, IV, V, X & $\mathrm{a}, \mathrm{c}, \mathrm{e}, \mathrm{i}$ \\
\hline \multirow[t]{4}{*}{ Osteichthyes } & Oncorhynchus mykiss & Naturalizada & 7 & $\mathrm{X}, \mathrm{XI}$ & $b, c, e, f, h$ \\
\hline & Oncorhynchus kisutch & Naturalizada & 5 & $\mathrm{X}, \mathrm{XI}$ & $\mathrm{c}, \mathrm{e}, \mathrm{h}$ \\
\hline & Oncorhynchus tshawytscha & Naturalizada & 1 & $\mathrm{X}, \mathrm{XI}$ & $\mathrm{h}$ \\
\hline & Salmo salar & Naturalizada & 6 & $\mathrm{X}, \mathrm{XI}$ & $\mathrm{c}, \mathrm{e}, \mathrm{f}, \mathrm{h}$ \\
\hline \multirow[t]{5}{*}{ Polychaeta } & Polydora uncinata & Naturalizada & 2 & IV & $\mathrm{a}$ \\
\hline & Polydora rickettsi & Naturalizada & 2 & $\mathrm{X}$ & d \\
\hline & Polydora bioccipitalis & Nativa $^{5}$ & 2 & II, IV & $\mathrm{a}$ \\
\hline & Dipolydora giardi & Naturalizada & 1 & $\mathrm{X}$ & $\mathrm{a}$ \\
\hline & Terebrasabella heterouncinata & Naturalizada & 2 & $\mathrm{~V}, \mathrm{X}$ & $\mathrm{a}$ \\
\hline \multirow[t]{2}{*}{ Bryozoa } & Bugula neritina & Naturalizada & 5 & IV & $\mathrm{c}, \mathrm{e}, \mathrm{g}$ \\
\hline & Bugula flabellata & Naturalizada & 2 & IV & $\mathrm{g}$ \\
\hline
\end{tabular}

${ }^{1}$ No sólo muestra las regiones administrativas donde se han llevado a cabo experimentos, sino también aquellas donde se han realizado búsquedas de la especie objetivo, donde no necesariamente se encuentra presente (ver Materiales y métodos).

${ }^{2}$ sensu Macaya et al. (2013)

${ }^{3}$ Identificada como Grateloupia intestinalis por Collantes \& Muñoz-Muga (2009)

${ }^{4}$ sensu Madariaga et al. (2014)

${ }^{5}$ Especie considerada exótica por Moreno et al. (2006), pero reconocida como nativa por Riascos et al. (2009) basado en análisis del registro fósil

${ }^{3}$ LIB. 2016. SCT № 20/2015 'Consultoría para elaborar el catálogo de las especies exóticas asilvestradas/naturalizadas en Chile, en el marco del Proyecto GEF/MMA/PNUD EEI AJF'. Informe Técnico. Laboratorio de Invasiones Biológicas, Universidad de Concepción, Concepción, Chile. 
La ascidia Pyura praeputialis (Heller, 1878) es la especie que ha recibido mayor atención en cuanto al número de trabajos publicados, siendo modelo de estudio para todas las temáticas analizadas (Tabla 1). Sin embargo, la distribución de esta especie en Chile se encuentra restringida a la Bahía de Antofagasta, lo que dificulta la extrapolación de dichos resultados al resto de la costa chilena. La macroalga Codium fragile subsp. fragile (Suringar) Hariot (antes C.fragile subsp. tomentosoides (van Goor) P.C.Silva) y el bivalvo Mytilus galloprovincialis (Lamarck, 1819) son otras dos especies con alto número de trabajos, pero sólo $C$. fragile ha sido modelo de estudio para una amplia variedad de temáticas (Tabla 1).

a)

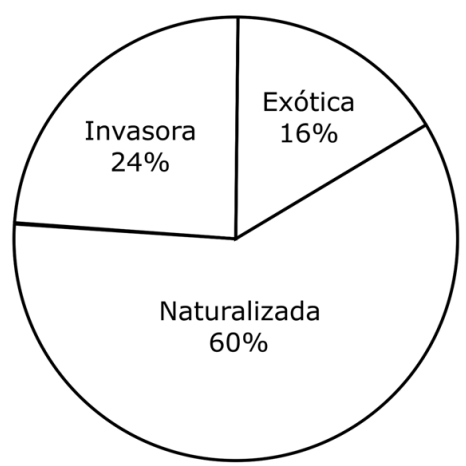

b)

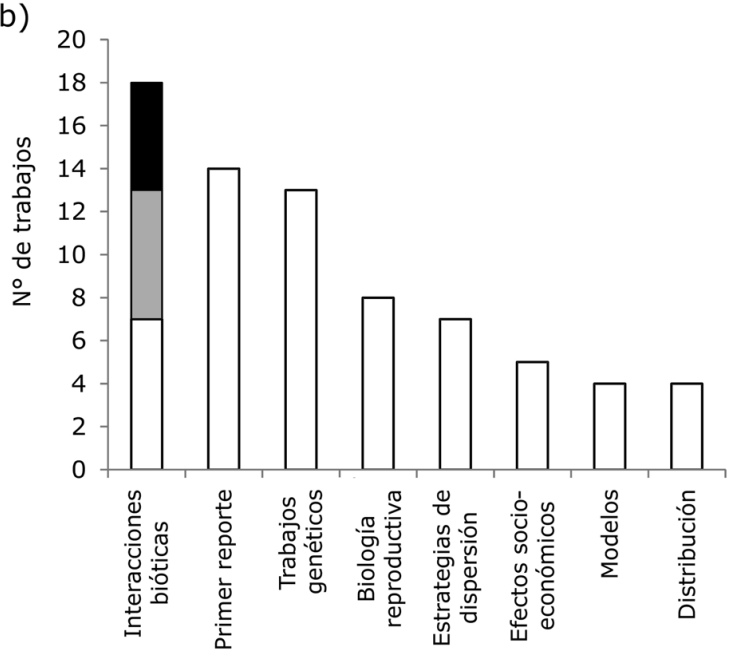

Figura 1. a) Porcentaje de trabajos relacionados con ecología de invasiones marinas en Chile clasificados según estatus de la especie introducida. b) Número de trabajos según temática abordada. En interacciones bióticas: blanco representa depredación, grisfacilitación y negro competencia / a) Percentage of papers related to ecology of marine invasions in Chile by the status of introduced species. b) Number of papers as topics addressed. In biotic interactions: predation in white, facilitation in gray, and competition in black
La mayoría de los artículos analizados (60\%) evalúan especies en etapa de naturalización (Fig. 1a), es decir que forman poblaciones estables sin intervención humana directa, pero nohay evidencia de una tendencia a invadir. Por otra parte, sólo un $24 \%$ de los artículos evalúan especies invasoras, lo que marca una clara diferencia con lo observado en sistemas terrestres en donde más del 50\% de las publicaciones tanto en plantas como animales tienen como objetivo a especies invasoras (Quiroz et al. 2009).

Según Castilla \& Neill (2009) la mayoría de las especies introducidas en la costa de Chile mantienen rangos acotados de distribución, y las únicas especies que se comportan como invasoras (i.e., expanden su rango de distribución en el área introducida) corresponden a la anémona criptogénica Anemonia alicemartinae (Häussermann \& Försterra, 2001) y la macroalga asiática Codium fragile, por lo que es esperable que la cantidad de publicaciones relacionadas con especies invasoras sea menor que las relacionadas con especies naturalizadas. No obstante, durante los últimos años se ha observado un incremento significativo en el rango de distribución de algunas especies exóticas que hasta ese entonces no se consideraban invasoras, como la ascidia Ciona robusta (Hoshino \& Tokioka, 1967) (antes Ciona intestinalis (Linnaeus, 1767)) (Madariaga et al. 2014), y las macroalgas Mastocarpus latissimus (Harvey) S.C. Lindstrom, Hughey \& Martone (Macaya et al. 2013), Schottera nicaeensis (J.V.Lamouroux ex Duby) Guiry \& Hollenberg (VillaseñorParada et al. 2014) y Asparagopsis armata Harvey (Ramírez et al. 2007). Por esta razón, una re-evaluación de las categorías asignadas a cada especie introducida (i.e., naturalizada, invasora) se hace necesaria, de acuerdo a los nuevos antecedentes que se puedan conocer respecto al comportamiento de estas especies en sistemas costeros chilenos.

\section{SíNTESIS DEL ANÁLISIS DE LA LITERATURA SELECCIONADA}

De los 71 trabajos seleccionados, 7 correspondían a revisiones y comentarios cuya finalidad era sintetizar información respecto a un amplio espectro de especies exóticas en sistemas marinos (e.g., Castilla et al. 2005, Camus 2005), o bien, en algunos taxa particulares (e.g., abalones, Godoy \& Jerez 1998, Flores-Aguilar et al. 2007; poliquetos perforadores, Moreno et al. 2006; salmones, Gajardo \& Laike 2003, García de Leaniz et al. 2010). El resto de los trabajos fueron agrupados en 6 categorías, respecto a los tópicos abordados (en donde un mismo trabajo podía abordar más de un tópico) (Fig. 1b, Tabla 1). 


\section{A) Primeros reportes}

La mayoría de los trabajos analizados correspondieron a reportes puntuales de la ocurrencia de una nueva especie, en algún lugar particular de la costa chilena. Estos reportes eran acompañados de una descripción morfológica, y en algunos casos estaban respaldados con análisis moleculares, que permitieron inferir el lugar de origen de dicha introducción.

La costa del Pacífico NO (i.e., sudeste asiático) ha sido identificada como el lugar de origen de varias especies exóticas en la costa chilena, como las macroalgas Polysiphonia morrowii Harvey (Kim et al. 2004), Scytosiphon tenellus Kogame (Camus et al. 2005) y Codium fragile (Provan et al. 2005). El Atlántico NE en cambio, ha sido identificado como el lugar de origen de la medusa Aurelia aurita (Linnaeus, 1758) (Häussermann et al. 2009).

La identificación de vectores de introducción, en cambio, ha sido menos evaluada, y sólo argumentada a partir de observaciones indirectas. Por ejemplo, la ocurrencia de los poliquetos perforadores exóticos Polydora uncinata (SatoOkoshi, 1998), Terebrasabella heterouncinata (Fitzhugh \& Rouse, 1999), Polydora rickettsi (Woodwick, 1961) y Dipolydora giardi (Mesnil, 1896) en conchas de abalones y ostras japonesas de sistemas de cultivos, ha permitido inferir una introducción de estas especies mediante acuicultura (Radashevsky \& Olivares 2005, Vargas et al. 2005, Moreno et al. 2006), mientras que, basado en la dinámica del tráfico marítimo en la Patagonia, se ha deducido una introducción de Aurelia aurita desde el Atlántico NE mediante aguas de lastre (Häussermann et al. 2009).

\section{B) Trabajos genéticos}

Una buena parte de los trabajos analizados incorporaban análisis genéticos. Algunos de estos trabajos reportaban por primera vez la especie en Chile y mediante la comparación de secuencias, identificaban el lugar desde donde fueron introducidas (ver ejemplos en la sección primeros reportes).

Estudios genéticos también han permitido aclarar la real identidad taxonómica de especies crípticas, por ejemplo, Tarifeño et al. (2012) a partir del uso de Polimorfismos de Longitud de Fragmentos de Restricción (RFLP) identificaron la presencia de la especie introducida Mytilus galloprovincialis (Lamarck 1819) en la costa de Concepción, la cual había sido erróneamente identificada como la especie nativa Mytilus chilensis (Hupe 1854).

Otros estudios genéticos han sido utilizados para caracterizar genética y/o fenotípicamente las poblaciones introducidas (e.g., Astorga et al. 2002), y detectar procesos de hibridación con especies nativas (e.g., Westfall \& Gardner 2013).

\section{c) Interacciones bióticas: Depredación}

Especies exóticas sirven como alimento para aves marinas, por ejemplo Pyura praeputialis en el norte de Chile (Pacheco \& Castilla 2000, 2001) y salmones en la costa sur (Jiménez et al. 2013).

En algunos casos, depredadores nativos pueden generar una importante resistencia biótica sirviendo como control biológico para evitar el crecimiento de especies exóticas dañinas, sobre implementos utilizados en el cultivo de moluscos (Dumont $e t$ al. 2009, 2011a, b). Esta resistencia biótica generada por depredadores bentónicos, junto a las características oceanográficas particulares de la costa chilena, han sido propuestas como factores que generan una barrera natural ante la invasión de nuevas especies (Castilla \& Neill 2009).

La evaluación de potenciales efectos que especies marinas introducidas, aún no naturalizadas, podrían generar ante un eventual escape (i.e., supervivencia en sistemas naturales de un organismo mantenido en cautiverio; Falk-Petersen et al. 2006) ha sido evaluada sólo para el abalón Haliotis discus hannai (Stotz et al. 2006). Esta especie es capaz de consumir hasta el $5 \%$ de su peso corporal desde una variada oferta de macroalgas, lo que constituye un importante riesgo para las comunidades de macroalgas nativas (Camus 2005, Stotz et al. 2006).

\section{D) Interacciones bióticas: Facilitación}

Interacciones positivas han sido observadas entre especies nativas y exóticas, por ejemplo, Pyura praeputialis genera una matriz que es utilizada como sustrato secundario para el asentamiento de numerosas especies bentónicas, lo que incrementa significativamente la diversidad local (Cerda \& Castilla 2001, Castilla et al. 2004a, 2014). Además, moluscos exóticos en sistemas de cultivo son infectados por poliquetos perforadores de conchas que utilizan a estas especies como nuevos sustratos de asentamiento (Vargas et al. 2005, Avilés et al. 2007a, b; Rozbaczylo et al. 2007).

Sin embargo, la facilitación no sólo puede ocurrir entre especies nativas y exóticas, sino además entre dos especies exóticas, por ejemplo, en el norte de Chile, la macroalga de origen asiático Neosiphonia harveyi (Bailey) M.-S. Kim, H.G. Choi, Guiry \& G.W. Saunders, es el epifito más conspicuo en la macroalga invasora Codium fragile, la que además de proporcionarle un sustrato secundario para su asentamiento, podría servirle como un potencial vector de dispersión secundaria (Villaseñor-Parada \& Neill 2011). 


\section{e) Interacciones bióticas: Competencia}

Competencia es la interacción biótica que ha sido menos evaluada en ecología de invasiones marinas en Chile (Fig. 1). Ocurrencia de competencia entre especies exóticas y nativas ha sido reportada entre salmones y la fauna íctica nativa (Soto et al. 2001), entre la ascidia exótica Pyura praeputialis y el mitilido nativo Perumytilus purpuratus (Lamarck, 1819) (Castilla et al. 2004b, Caro et al. 2011), y entre el briozoo Bugula neritina (Linnaeus, 1758) y especies incrustantes en sistemas artificiales (Cifuentes et al. 2010).

Potencial competencia entre el abalón Haliotis discus hannai y herbívoros nativos ha sido evaluada en laboratorio, ante un eventual escenario de escape desde centros de cultivo (Stotz et al. 2006).

\section{F) Biología ReProductiva}

Varios trabajos describen aspectos reproductivos y de biología del desarrollo de especies exóticas marinas, algunos en comparaciones con especies nativas (e.g., Toro et al. 2012, González et al. 2014, Oyarzún et al. 2014), y otros han sido utilizados para explicar estrategias de dispersión (mencionadas más adelante).

\section{G) ESTRategias DE DISPERSIÓN}

Diferentes métodos de dispersión han sido identificados para especies exóticas entre los que se incluyen la adhesión a estructuras antrópicas y mecanismos alternativos de reproducción (usualmente de tipo asexual).

La anémona Anemonia alicemartinae, si bien es una especie dioica, no se han encontrado machos maduros, por lo que, al parecer, su reproducción es exclusivamente asexual mediante fisión binaria (Häussermann \& Försterra 2001). López et al. (2013) proponen como mecanismo de dispersión una estrategia de 'desprendimiento y re-adhesión' basada en el desprendimiento desde el sustrato de individuos que, luego de quedar a la deriva, se re-adhieren en localidades cercanas, permitiéndoles colonizar paulatinamente nuevos sitios.

Para la macroalga Codium fragile, se han descrito 2 estrategias de dispersión: a) fragmentación y liberación de talos a la deriva que le permitiría a las zonas más reproductivas del talo (i.e., sección apical) llegar a nuevos sitios y liberar gametos que, mediante partenogénesis, podrían dar origen a nuevos individuos (Villaseñor-Parada et al. 2013); y b) liberación de utrículos aislados, los que, mediante reproducción vegetativa, podrían dar origen a nuevos individuos, aún más rápido que su congénere nativa Codium bernabei A.V.González, M.E.Chacana \& P.C.Silva (González et al. 2014).
La macroalga roja Mastocarpus latissimus, también ha mostrado un incremento significativo en su rango de distribución a través del tiempo (Macaya et al. 2013), lo que podría verse favorecido por el desprendimiento de talos reproductivos y liberación de papilas (estructuras que contienen cistocarpos) a la deriva (Oróstica et al. 2012).

En el norte de Chile se ha observado el importante rol de boyas a la deriva, como mecanismo efectivo de dispersión a larga distancia para algunos invertebrados introducidos como Ciona robusta o Bugula neritina (Astudillo et al. 2009). En el sur de Chile, Manríquez et al. (2014) han estudiado las comunidades incrustantes en pilotes de muelles en sectores con alto tráfico marítimo. Si bien no encontraron presencia de especies introducidas, dicho trabajo entrega resultados novedosos y muy útiles para ayudar a dilucidar el rol potencial de estructuras antrópicas como lugar de refugio para estas especies, a la espera de condiciones óptimas para el establecimiento en sistemas naturales.

Por último, en el norte de Chile se ha observado cómo el asentamiento de individuos juveniles de la ascidia Pyura praeputialis ocurre generalmente en las periferias de colonias pre existentes, lo que podría constituir un mecanismo de auto facilitación intraespecífica (Alvarado et al. 2001).

\section{н) Efectos socio-económicos}

Algunos trabajos han evaluado el efecto directo de especies exóticas sobre actividades económicas, como por ejemplo, la disminución del rendimiento en el cultivo de Gracilaria spp. en el norte de Chile con el incremento en la abundancia de Codium fragile, que provoca un enlentecimiento en el proceso de extracción al invertir tiempo en desprender ambas macroalgas (Neill et al. 2006).

Otros trabajos en cambio, han evaluado la efectividad de mecanismos para contrarrestar los efectos negativos de las especies invasoras, principalmente de aquellas que son parte de las comunidades incrustantes que sobrecrecen en sistemas de cultivo, como Ciona robusta o Pyura praeputialis. Algunos de estos mecanismos incluyen la aplicación de sustancias antifouling derivadas de cultivos de bacterias (Zapata et al. 2007) y el uso de depredadores bentónicos como controladores biológicos (Dumont et al. 2011a, b).

\section{I) Modelos}

Algunos trabajos han aplicado modelos de nicho ecológico para identificar hábitats más susceptibles a ser invadidos, ante una eventual expansión de especies con alto potencial invasor a nivel mundial, como Crassostrea gigas, Ciona robusta y 
Codium fragile (Carrasco \& Baron 2010, Madariaga et al. 2014). Estos trabajos muestran la alta vulnerabilidad de gran parte de la costa chilena, ante la invasión de dichas especies.

Otros trabajos evalúan la sustentabilidad de los cultivos de abalones mediante la aplicación de modelos eco-sociales (Ortiz \& Stotz 2007), y el 'auto-raleo' en mantos del tunicado Pyura praeputialis mediante modelos morfométricos (Guiñez \& Castilla et al. 2001).

\section{J) Distribución en Chile}

La distribución a lo largo de la costa chilena ha sido descrita sólo para 4 especies exóticas: Anemonia alicemartinae (Häusermann \& Försterra 2001), Mytilus galloprovincialis (Toro et al. 2005), Codium fragile (Neill et al. 2006), y Mastocarpus latissimus (Macaya et al. 2013).

\section{ANÁLISIS CUANTITATIVo de LA LITERATURA RELACIONADA con ecología de invasiones marinas en ChILE}

\section{a) Tendencia temporal del estudio de ecología de inVASIONES MARINAS EN ChILE}

El número de artículos por año incrementa significativamente con el tiempo (Fig. 2), sugiriendo un creciente interés en investigación relacionada con invasiones marinas. Sin embargo, este incremento pareciera ser más bien lineal y no exponencial como se ha observado en sistemas terrestres de Chile (Quiroz et al. 2009), sistemas marinos a nivel mundial (Davidson \& Simkanin 2011), o sistemas terrestres y dulceacuícolas a nivel mundial (Puth \& Post 2005); quizás porque el desarrollo de la ecología de invasiones marinas en Chile es incipiente comparado con los otros casos.

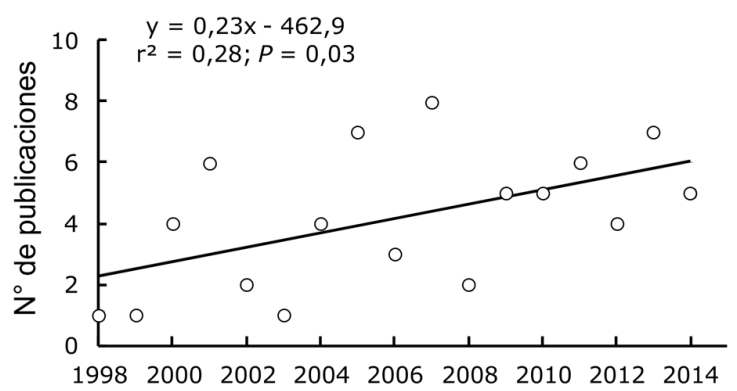

Figura 2. Número de publicaciones relacionadas con ecología de invasiones marinas en Chile a través del tiempo (1998-2014). Se muestra además el resultado del análisis de regresión lineal / Number of papers related to ecology of marine invasions in Chile over time (1998-2014). The result of the linear regression analysis is also shown

\section{B) Distribución espacial de la información DISPONIBLE}

La cantidad de investigaciones realizadas no es homogénea a lo largo de Chile (Fig. 3a). Un alto número de investigaciones han sido realizadas en localidades de las regiones de Antofagasta y Los Lagos (19 y 15 trabajos respectivamente), mientras que otras regiones como Arica y Tarapacá han sido escasamente estudiadas.

Muy pocas investigaciones se han realizado en las regiones de Valparaíso y Magallanes, las que se caracterizan por un alto tráfico marítimo en sus costas (el que suele asociarse a una alta intensidad en la presión de propágulos, Hewitt et al. 2009).

Además, si consideramos los grupos taxonómicos sobre los cuales se han realizado los trabajos, existe una tendencia a investigar el mismo grupo taxonómico (y usualmente la misma especie) por región (Fig. 3b). Por ejemplo, a pesar de ser Antofagasta la región más estudiada, los trabajos están sesgados hacia un grupo en particular (i.e., ascidias, Fig. 3b) y a pesar que se han estudiado cuatro de estas especies, casi la totalidad de dichos trabajos han sido realizados en sólo una: Pyura praeputialis. De la misma manera, en la región del Biobío, la tercera con más publicaciones a nivel nacional, el 60\% de ellas corresponden a estudios sobre Mytilus galloprovincialis (Fig. 3b). Esta misma tendencia es observada en casi todo el país, a excepción de las regiones de Coquimbo y Los Lagos, en donde no sólo se estudia equitativamente una amplia variedad de grupos taxonómicos, sino que además, son las regiones en las que se estudia la mayor cantidad de especies introducidas (7 especies en Coquimbo y 14 especies en Los Lagos, Fig. 3b).

Por último, una tendencia observada en casi todas las regiones fue el fuerte 'centralismo' en las investigaciones. A excepción de trabajos que buscaban identificar la distribución de especies exóticas a lo largo de la costa chilena, la mayoría de las investigaciones eran realizadas en los mismos sectores, usualmente cercanos a la capital regional o a sedes universitarias (e.g., Bahía de Antofagasta en Antofagasta, Bahía Calderilla en Atacama, Bahía La Herradura en Coquimbo, Bahía de Concepción en el Biobío, Mar interior de Chiloé y Seno de Reloncaví en Los Lagos, Estrecho de Magallanes en Magallanes).

Basado en los avances en cuanto a taxonomía y sistemática, la problemática de acceso y muestreo a una mayor variedad de hábitats marinos, y la disponibilidad de registros históricos, Carlton (2009) considera que la cantidad de especies exóticas reportadas para la costa chilena por Castilla et al. (2005) y Castilla \& Neill (2009) se encuentra subestimada, siendo entre cinco y diez veces más. En este trabajo, la desigual cantidad de información a lo largo del país, el sesgo hacia una especie o 

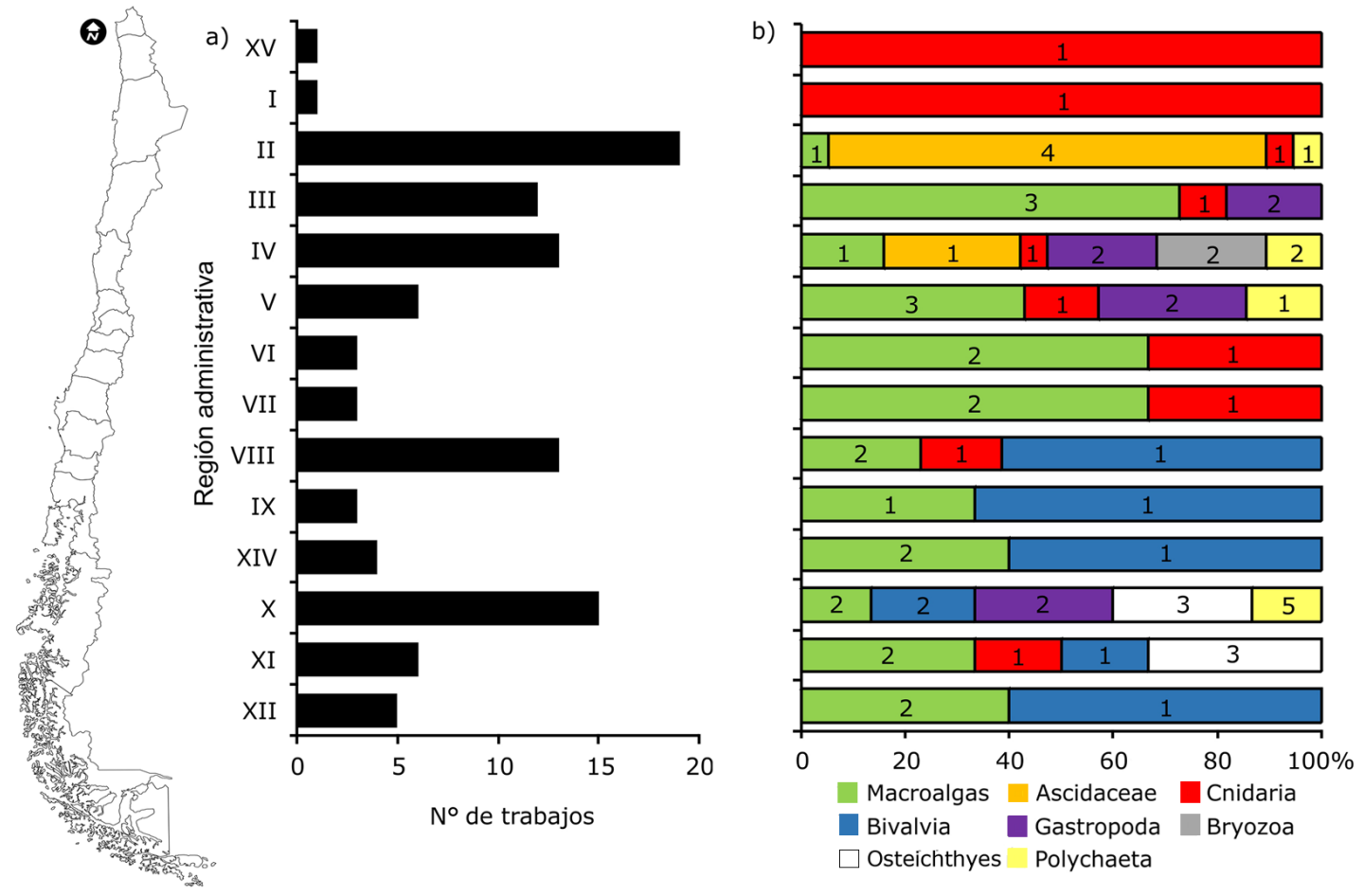

Figura 3. a) Número de trabajos realizados por región político-administrativa. b) porcentaje de trabajos por taxa realizado en cada región. Números arábigos sobre las barras indican el número de especies exóticas estudiadas para cada taxa en cada región. Un mismo trabajo podía abarcar más de una región administrativa y más de un taxa. XV=Región de Arica y Parinacota, I=Región de Tarapacá, II=Región de Antofagasta, III=Región de Atacama, IV=Región de Coquimbo, V=Región de Valparaíso, VI=Región de O'Higgins, VII=Región del Maule, VIII=Región del Biobío, IX=Región de la Araucanía, $\mathbf{X I V = R e g i o ́ n ~ d e ~ L o s ~ R i ́ o s , ~} \mathbf{X = R e g i o ́ n ~ d e ~ L o s ~ L a g o s , ~} \mathbf{X I = R e g i o ́ n ~ d e ~ A y s e ́ n ~ y ~ X I I = R e g i o ́ n ~ d e ~ M a g a l l a n e s ~ / ~ a ) ~ N u m b e r ~ o f ~ p a p e r s ~ w r i t t e n ~ b y ~ g e o p o l i t i c a l ~ r e g i o n . ~}$ b) percentage of papers for taxa held on each geopolitical region. Arabic numerals on the bars indicate the number of exotic species for each taxa studied on each region. One same work could cover more than one geopolitical region and more than one taxa. XV= Arica and Parinacota Region, I= Tarapacá Region, II= Antofagasta Region, III= Atacama Region, IV= Coquimbo Region, V= Valparaíso Region, VI= O’Higgins Region, VII= Maule Region, VIII= Biobío Region, IX= Araucanía Region, XIV= Los Ríos Region, X= Los Lagos Region, XI= Aysén Region and XII= Magallanes Region

grupo taxonómico particular, y el 'centralismo' dentro de las regiones administrativas, constituyen evidencia de falta de información respecto a las especies exóticas en la costa de Chile continental. Considerando que la cantidad de información disponible se relaciona directamente con la cantidad de reportes de especies exóticas (McGeoch et al. 2010), este trabajo entrega evidencia que apoya la hipótesis que la baja cantidad de reportes de especies exóticas en sistemas marinos chilenos se debe más bien a un artefacto (producto de la heterogeneidad de cantidad de información disponible) que a una resistencia natural de la costa chilena ante la invasión.

\section{C) Escala ESPaCial y temporal abarcada en los ESTUDIOS}

En ecología de invasiones, al igual que en el resto de las subdisciplinas de la ecología, la escala (tanto espacial como temporal) en la cual se desarrollan las investigaciones, cobra gran importancia en la interpretación y extrapolación de los resultados obtenidos (Davis et al. 2000, Pauchard \& Shea 2006, Stachowicz \& Byrnes 2006).

Los cambios en la escala espacial, se asocian a un aumento en la heterogeneidad ambiental, afectando diversos procesos (e.g., dispersión, relación diversidad exótica $v s$. diversidad nativa, control, impacto) que son claves para entender el fenómeno de la invasión (Pauchard \& Shea 2006). Respecto a la escala espacial abarcada en las investigaciones realizadas en la costa de Chile continental, la gran mayoría de los trabajos fueron realizados en una sola región (Fig. 4a), en uno o más sitios (usualmente cercanos). En general, las investigaciones abarcaban ciertos sectores de la costa (cercanos a la capital regional o a centros universitarios), tal como se describe en la sección anterior. Cuatro trabajos abarcaron 2 regiones 

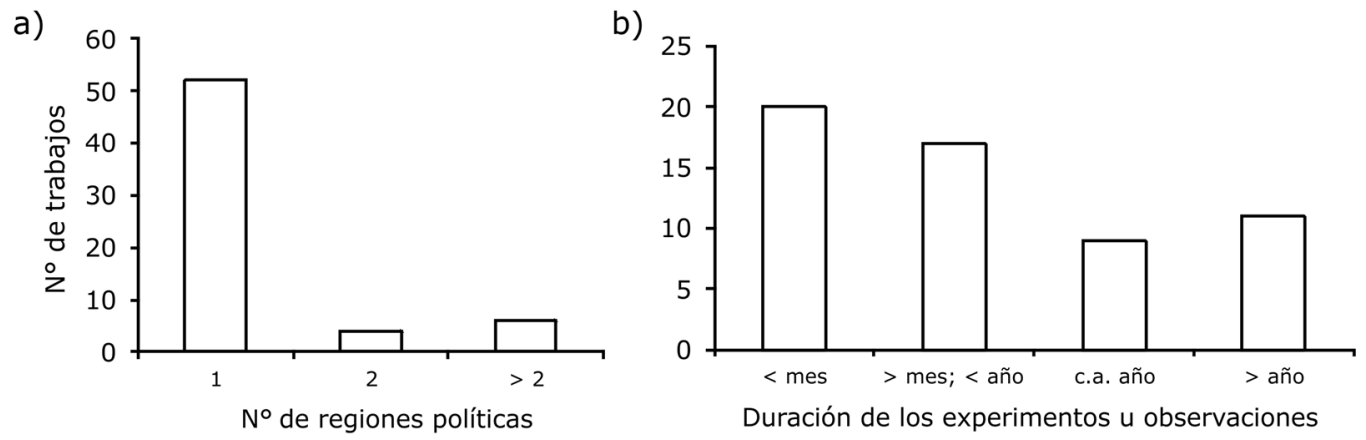

Figura 4. a) Número de regiones administrativas abarcadas. b) Duración de los experimentos u observaciones, en los trabajos analizados / Number of administrative regions covered. b) Time length of experiments or observations in the analyzed papers

(usualmente contiguas) y solo los trabajos cuyo objetivo fue evaluar la distribución de especies exóticas a lo largo de Chile, abarcaron escalas espaciales más grandes ( $>2$ regiones).

Por otra parte, el incremento en la escala temporal permite evaluar el comportamiento de la especie con los cambios en la disponibilidad de recursos y la dinámica de las poblaciones nativas, que por lo general no son constantes en el tiempo (Davis et al. 2000, Stachowicz \& Byrnes 2006). En cuanto a la escala temporal abarcada en los trabajos analizados, los resultados son más homogéneos, ya que, si bien la mayoría de las investigaciones duraron menos de un mes, un buen número de ellas abarcaban estudios estacionales, anuales e interanuales (Fig. 4b).

Por último, trabajos que evalúen fenómenos a múltiples escalas entregan resultados novedosos que permiten descubrir propiedades emergentes asociadas a estos cambios de escala (Pauchard \& Shea 2006). Sin embargo, ninguno de los trabajos analizados evaluó patrones a diferentes escalas.

\section{Conclusiones}

Uno de las principales tendencias observadas en esta revisión, es el creciente número de reportes de especies exóticas en la costa de Chile continental. El uso de herramientas moleculares en la identificación de estas especies (e.g., 'DNA barcoding') ha permitido grandes avances, incluso en la identificación del origen de la introducción, y su potencial vector. El uso de estos análisis ha permitido corroborar la identidad taxonómica de especies exóticas, por ejemplo Collantes \& Muñoz-Muga (2009) reportaron la introducción de la macroalga roja Grateloupia intestinalis (Harvey) Setchell ex P.G. Parkinson en un sector muy puntual de la Bahía de Valparaíso, pero análisis moleculares han determinado que corresponde a
Glaphyrosiphon chilensis M.E. Ramírez, Leister \& P.W. Gabrielson, especie que se había descrito previamente para la costa de la región de Los Ríos (P. Muñoz-Muga, com. pers.) $)^{4}$. Los avances en estas materias son muy importantes en la actualización de catastros de especies exóticas ya existentes.

En cuanto a la distribución de las especies exóticas a lo largo de la costa chilena, aún no es del todo clara, no sólo por la falta de trabajos dedicados a determinar su distribución, sino además, por el difícil acceso a ciertos sectores (e.g., zona de los canales y fiordos en el extremo sur). Esto ha llevado a que los catastros de especies exóticas, incluso los más actualizados (e.g., LIB $2016)^{3}$ infieran la distribución de estas especies en base a revisiones de artículos publicados o bien muestras conservadas en herbarios y/o museos. Si bien las colecciones de museos han sido reconocidas como herramientas eficaces para el estudio de la biodiversidad en general (Ramírez 2001) e invasiones biológicas en particular (Fuentes et al. 2013), el uso exclusivo de este tipo de información puede sesgar la información hacia zonas más estudiadas, lo que presenta una desventaja frente a muestreos in situ. Observaciones periódicas en terreno, sobre todo en zonas muy intervenidas como los alrededores de centros portuarios y/o acuícolas, podrían entregar información útil que permitiría actualizar la información de catastros previos. Por ejemplo, algunas especies exóticas como la ascidiaAsterocarpa humilis (Heller, 1878) y el briozoo Bugula flabellata (Thompson in Gray 1848), cuya distribución se creía restringida a la costa norte del país (Clarke \& Castilla 2000, Astudillo et al. 2009), han sido encontradas varios kilómetros más al sur, adheridas a barcos y sustratos artificiales, en el sector portuario de la Bahía de Concepción (Cancino \& Gallardo 2004, Pinochet 2016).

${ }^{4}<$ pilar.munoz@cienciasdelmar.cl> 
La falta de información respecto a la distribución de las especies exóticas, y su tasa de expansión en la costa chilena, influye también en la categorización de las especies introducidas (e.g., naturalizada, invasora). Tradicionalmente sólo dos especies, Codium fragile y Anemonia alicemartinae, eran consideradas invasoras por expandir su rango de distribución tras su introducción en la costa chilena (Castilla et al. 2005, Castilla \& Neill 2009). Sin embargo, trabajos más recientes han detectado un aumento en el rango de distribución de varias especies exóticas cuya distribución se creía acotada a ciertos sectores de la costa (e.g., Asparagopsis armata, Ramírez et al. 2007; Mastocarpus latissimus, Macaya et al. 2013; Schottera nicaeensis, Villaseñor-Parada et al. 2014; Ciona robusta, Madariaga et al. 2014), lo que indica un comportamiento invasor en estas especies. Observaciones periódicas en la costa chilena podrían detectar otras especies con un comportamiento similar.

Por último, la cantidad de información, desigual a nivel nacional y sectorial a nivel regional, y con sesgos hacia ciertos grupos taxonómicos, constituye un fuerte argumento para apoyar la idea de una subestimación del número de especies exóticas en la costa de Chile continental. Estudios basados en cuidadosos trabajos taxonómicos y sistemáticos, exhaustivas recopilaciones de datos históricos y acuciosas observaciones en terreno, permitieron cuadruplicar el número de especies exóticas en la costa de Sudáfrica, la cual se creía una de las más prístinas a nivel mundial (Mead et al. 2011). Un análisis de este tipo en las costas chilenas podría entregar interesante información al respecto.

\section{Agradecimientos}

Todos los autores agradecen los comentarios de revisores anónimos que permitieron mejorar significativamente el manuscrito original. Este trabajo forma parte de la tesis para optar al grado de Doctor en Ciencias Biológicas área Botánica de C V-P quien es financiado por Beca CONICYT para doctorado nacional $\mathrm{N}^{\circ} 21110927$. ECM y CV-P agradecen el financiamiento de CONICYT a través de su programa FONDAP, Proyecto N ${ }^{\circ} 15150003$. AP y CV-P agradecen el financiamiento del Instituto de Ecología y Biodiversidad mediante los proyectos ICM P05-002 y CONICYT PFB-23.

\section{LITERATURA CITADA}

Alvarado JL, R Pinto, P Marquet, C Pacheco, R Guiñez \& JC Castilla. 2001. Patch recolonization by the tunicate Pyura praeputialis in the rocky intertidal of the Bay of Antofagasta, Chile: evidence for self-facilitation mechanisms. Marine Ecology Progress Series 224: 93-101.
Ambrogi OA. 2002. Current status of aquatic introductions in Italy. In: Leppäkoski E, S Gollasch \& S Olenin (eds). Invasive aquatic species of Europe. Distribution, impacts and management, pp. 311-324. Kluwer Academic Publishers, Dordrecht.

Astorga M, R Guiñez, JC Ortiz \& JC Castilla. 2002. Phenotypic and genetic variation in tunicate Pyura praeputialis (Heller, 1878) in the northern sac of the Antofagasta Bay. Revista Chilena de Historia Natural 75: 515-526.

Astudillo JC, M Bravo, CP Dumont \& M Thiel. 2009. Detached aquaculture buoys in the SE Pacific: a potential dispersal vehicle for associated organisms. Aquatic Biology 5:219-231.

Avilés F, N Rozbaczylo, M Herve \& M Godoy. 2007a. First report of polychaetes from the genus Oriopsis (Polychaeta: Sabellidae) associated with the Japanese abalone Haliotis discus hannai and other native molluscs in Chile. Journal of Shellfish Research 26(3): 863-867.

Avilés F, N Rozbaczylo, M Godoy \& G Muñoz. 2007b. The first report of Phoronis sp. (Phoronida) in red abalone (Haliotis rufescens) in Chile. Journal of Shellfish Research 26: 859-861.

Blackburn TM, P Pyšek, S Bacher, JT Carlton, RP Duncan, V Jarošík, JRU Wilson \& DM Richardson. 2011. A proposed unified framework for biological invasions. Trends in Ecology \& Evolution 26(7): 333-339.

Camus PA. 2005. Introducción de especies en ambientes marinos chilenos: no solo exóticas, no siempre evidentes. Revista Chilena de Historia Natural 78: 155-159.

Camus C, AP Meynard, S Faugeron, K Kogame \& JA Correa. 2005. Differential life history phase expression in two coexisting species of Scytosiphon (Phaeophyceae) in northern Chile. Journal of Phycology 41(5): 931-941.

Cancino JM \& JA Gallardo. 2004. Efectos del retardo del asentamiento en las expectativas de vida del briozoo Bugula flabellata (Bryozoa: Gymnolaemata). Revista Chilena de Historia Natural 77(2): 227-234.

Carlton JT. 1996. Biological invasions and cryptogenic species. Ecology 77(6): 1653-1655.

Carlton JT. 2009. Deep invasion ecology and the assembly of communities in historical time. In: Rilov G \& JA Crooks (eds). Marine bioinvasions: ecology, conservation, and management perspectives, pp. 439-457. Springer-Verlag, Berlin.

Caro AU, R Guiñez, V Ortiz \& JC Castilla. 2011. Competition between a native mussel and a non-indigenous invader for primary space on intertidal rocky shores in Chile. Marine Ecology Progress Series 428: 177-185.

Carrasco MF \& PJ Baron. 2010. Analysis of the potential geographic range of the Pacific oyster Crassostrea gigas (Thunberg, 1793) based on surface seawater temperature satellite data and climate charts: the coast of South America as a study case. Biological Invasions 12(8): 2597-2607. 
Castilla JC \& PE Neill. 2009. Marine bioinvasions in the Southeastern Pacific: status, ecology, economic impacts, conservation and management. In: Rilov G \& JA Crooks (eds). Marine bioinvasions: ecology, conservation, and management perspectives, pp. 439-457. Springer-Verlag, Berlin.

Castilla JC, N Lagos \& M Cerda. 2004a. Marine ecosystem engineering by the alien ascidian Pyura praeputialis on a mid-intertidal rocky shore. Marine Ecology Progress Series 268: $119-130$.

Castilla JC, R Guiñez, AU Caro \& V Ortiz. 2004b. Invasion of a rocky intertidal shore by the tunicate Pyura praeputialis in the Bay of Antofagasta, Chile. Proceedings of the National Academy of Sciences of the United States of America 101(23): 8517-8524.

Castilla JC, M Uribe, N Bahamonde, M Clarke, R Desqueyroux-Faúndez, H Moyano, N Rozbaczylo, B Santelices, C Valdovinos \& P Zavala. 2005. Down under the southeastern Pacific: marine non-indigenous species in Chile. Biological Invasions 7(2): 213-232.

Cerda M \& JC Castilla. 2001. Diversity and biomass of macroinvertebrates in intertidal matrices of the tunicate Pyura praeputialis (Heller, 1878) in the Bay of Antofagasta, Chile. Revista Chilena de Historia Natural 74: 841-853.

Cifuentes M, I Krueger, CP Dumont, M Lenz \& M Thiel. 2010. Does primary colonization or community structure determine the succession of fouling communities? Journal of Experimental Marine Biology and Ecology 395(1): 10-20.

Clarke M \& JC Castilla. 2000. Dos nuevos registros de ascidias (Tunicata: Ascidiacea) para la costa continental de Chile. Revista Chilena de Historia Natural 73(3): 503-510.

Collantes G \& P Muñoz-Muga. 2009. Massive proliferation of Grateloupia intestinalis (Hooker fil. et Harvey) Setchell ex Parkinson (Rhodophyta, Halymeniaceae), a non-native species in Valparaíso Bay, central Chile. Revista de Biología Marina y Oceanografía 44(2): 527-532.

Cranfield HJ, DP Gordon, RC Willan, BA Marshall, CN Battershill, MP Francis, WA Nelson, CJ Glasby \& GB Read. 1998. Adventive species in New Zealand. NIWA Technical Report 34: 1-48. <http://docs.niwa.co.nz/library/ public/NIWAtr34.pdf>

Davidson I \& C Simkanin. 2012. The biology of ballast water 25 years later. Biological Invasions 14: 9-13.

Davis MA, JP Grime \& K Thompson. 2000. Fluctuating resources in plant communities: a general theory of invasibility. Journal of Ecology 88(3): 528-534.

Dumont CP, JD Urriago, A Abarca, CF Gaymer \& M Thiel. 2009. The native rock shrimp Rhynchocinetes typus as a biological control of fouling in suspended scallop cultures. Aquaculture 292: 74-79.

Dumont CP, LG Harris \& CF Gaymer. 2011a. Anthropogenic structures as a spatial refuge from predation for the invasive bryozoan Bugula neritina. Marine Ecology Progress Series 427: $95-103$
Dumont CP, CF Gaymer \& M Thiel. 2011b. Predation contributes to invasion resistance of benthic communities against the non-indigenous tunicate Ciona intestinalis. Biological Invasions 13(9): 2023-2034.

Falk-Petersen J, T Bøhn \& OT Sandlund. 2006. On the numerous concepts in invasion biology. Biological Invasions 8(6): 1409-1424.

Flores-Aguilar RA, A Gutierrez, A Ellwanger \& R SearcyBernal. 2007. Development and current status of abalone aquaculture in Chile. Journal of Shellfish Research 26(3): 705-711.

Fuentes N, A Pauchard, P Sánchez, J Esquivel \& A Marticorena. 2013. A new comprehensive database of alien plant species in Chile based on herbarium records. Biological Invasions 15(4): 847-858.

Gajardo G \& L Laikre. 2003. Chilean aquaculture boom is based on exotic salmon resources: a conservation paradox. Conservation Biology 17: 1173-1174.

García de Leaniz CC, G Gajardo \& S Consuegra. 2010. From Best to Pest: changing perspectives on the impact of exotic salmonids in the southern hemisphere. Systematics and Biodiversity 8(4): 447-459.

Godoy C \& G Jerez. 1998. The introduction of abalone in Chile: ten years later. Journal of Shellfish Research 17(3): 603-605.

González AV, J Beltrán \& B Santelices. 2014. Colonisation and growth strategies in two Codium species (Bryopsidales, Chlorophyta) with different thallus forms. Phycologia 53(4): 353-358.

Goulletquer P, G Bachelet, P Guy-Sauriau \& P Noel. 2002. Open Atlantic coast of Europe- A century of introduced species into French waters. In: Leppäkoski E, S Gollasch \& $\mathrm{S}$ Olenin (eds). Invasive aquatic species of Europe. Distribution, impacts and management, pp. 276-290. Kluwer Academic Publishers, Dordrecht.

Guiñez R \& JC Castilla. 2001. An allometric tridimensional model of selfthinning for a gregarious tunicate. Ecology 82(8): 2331-2341.

Häussermann V \& G Försterra. 2001. A new species of sea anemone from Chile, Anemonia alicemartinae n. sp. (Cnidaria: Anthozoa). An invader or an indicator for environmental change in shallow water? Organisms Diversity Evolution 1:211-224.

Häussermann V, MN Dawson \& G Försterra. 2009. First record of the moon jellyfish, Aurelia for Chile. Spixiana 32(1): 3-7.

Hewitt CL, ML Campbell, RE Thresher, RB Martin, S Boyd, BF Cohen, DR Currie, MF Gomon, MJ Keough, JA Lewis, MM Lockett, N Mays, MA McArthur, TD O'Hara, GCB Poore, DJ Ross, MJ Storey, JE Watson \& RS Wilson. 2004. Introduced and cryptogenic species in Port Phillip Bay, Victoria, Australia. Marine Biology 144: 183-202. 
Hewitt CL, S Gollasch \& D Minchin. 2009. The vessel as vector: biofouling, ballast water and sediments. In: Rilov G \& JA Crooks (eds). Biological invasions in marine ecosystems: Ecological, management, and geographic perspectives, pp. 117-131. Ecological Studies Series. SpringerVerlag, Berlin.

Jiménez JE, AM Arriagada, FE Fontúrbel, PA Camus \& MI Ávila-Thieme. 2013. Effects of exotic fish farms on bird communities in lake and marine ecosystems. Naturwissenschaften 100(8): 779-787.

Kim MS, EC Yang, A Mansilla \& SM Boo. 2004. Recent introduction of Polysiphonia morrowii (Ceramiales, Rhodophyta) to Punta Arenas, Chile. Botanica Marina 47(5): 389-394.

Kimbro DL, BS Cheng \& ED Grosholz. 2013. Biotic resistance in marine environments. Ecology Letters 16(6): 821-833.

Lockwood JL, MF Hoopes \& MP Marchetti. 2007. Invasion ecology, 304 pp. Blackwell Publishers, Malden.

López DN, PA Arancibia \& PE Neill. 2013. Potential dispersal mechanisms of the cryptogenic anemone, Anemonia alicemartinae. Revista Chilena de Historia Natural 86(3): 369-372.

Macaya EC, S Pacheco, A Cáceres \& S Musleh. 2013. Range extension of the non-indigenous alga Mastocarpus sp. along the Southeastern Pacific coast. Revista de Biología Marina y Oceanografía 48(3): 661-665.

Madariaga DJ, MM Rivadeneira, F Tala \& M Thiel. 2014. Environmental tolerance of the two invasive species Ciona intestinalis and Codium fragile: their invasion potential along a temperate coast. Biological Invasions 16(12): 2507-2527.

Manríquez PH, E Fica, V Ortiz \& JC Castilla. 2014. Bioincrustantes marinos en el canal de Chacao, Chile: un estudio sobre potenciales interacciones con estructuras manufacturadas por el hombre. Revista de Biología Marina y Oceanografía 49(2): 243-265.

McGeoch M, SHM Butchart, D Spear, E Marais, EJ Kleynhans, A Symes, J Chanson \& M Hoffmann. 2010. Global indicators of biological invasions: species numbers, biodiversity impact and policy responses. Diversity and Distributions 16:95-108.

Mead A, JT Carlton, CL Griffiths \& M Rius. 2011. Revealing the scale of marine bioinvasions in developing regions: a South African re-assessment. Biological Invasions 13(9): 1991-2008.

Moreno RA, PE Neill \& N Rozbaczylo. 2006. Native and non-indigenous boring polychaetes in Chile: a threat to native and commercial mollusk species. Revista Chilena de Historia Natural 79: 263-278.

Neill PE, O Alcalde, S Faugeron, SA Navarrete \& JA Correa. 2006. Invasion of Codium fragile spp. tomentosoides in northern Chile: a new threat for Gracilaria farming. Aquaculture 259: 202-210.
Nuñez MA \& A Pauchard. 2010. Biological invasions in developing and developed countries: does one model fit all? Biological Invasions 12(4): 707-714.

Oróstica MH, RD Otaíza \& PE Neill. 2012. Blades and papillae as likely dispersing propagules in Chilean populations of Mastocarpus sp. (Rhodophyta, Gigartinales). Revista de Biología Marina y Oceanografía 47(1): 109-119.

Ortiz M \& W Stotz. 2007. Ecological and eco-social models for the introduction of the abalone Haliotis discus hannai into benthic systems of north-central Chile: sustainability assessment. Aquatic Conservation: Marine and Freshwater Ecosystems 17(1): 89-105.

Oyarzún PA, JE Toro, O Garrido, C Briones \& R Guiñez. 2014. Differences in sperm ultrastructure between Mytilus chilensis and Mytilus galloprovincialis (Bivalvia, Mytilidae): could be used as a taxonomic trait? Latin American Journal of Aquatic Research 42(1): 172-179.

Pacheco CJ \& JC Castilla. 2000. Ecología trófica de los ostreros Haematopus palliatus pitanay (Murphy 1925) y Haematopus ater (Vieillot et Oudart 1825) en mantos del tunicado Pyura praeputialis (Heller 1878) en la Bahía de Antofagasta, Chile. Revista Chilena de Historia Natural 73: 533-542.

Pacheco CJ \& JC Castilla. 2001. Foraging behavior of the American oystercatcher Haematopus palliatus pitanay (Murphy 1925) on the intertidal ascidian Pyura praeputialis (Heller 1878) in the Bay of Antofagasta, Chile. Journal of Ethology 19(1): 23-26.

Pauchard A \& K Shea. 2006. Integrating the study of nonnative plant invasions across spatial scales. Biological Invasions 8(3): 399-413.

Pinochet J. 2016. Cascos de los buques como vectores para la introducción de especies marinas no-indígenas en el puerto de Talcahuano, Chile. Tesis de Magister, Facultad de Ciencias, Universidad Católica de la Santísima Concepción, Concepción, 21 pp.

Provan JIM, S Murphy \& CA Maggs. 2005. Tracking the invasive history of the green alga Codium fragile spp. tomentosoides. Molecular Ecology 14(1): 189-194.

Puth LM \& DM Post. 2005. Studying invasion: have we missed the boat? Ecology Letters 8: 715-721.

Pyšek P, DM Richardson, J Pergl, V Jarošík, Z Sixtová \& E Weber. 2008. Geographical and taxonomic biases in invasion ecology. Trends in Ecology \& Evolution 23(5): 237 244.

Quiroz CL, A Pauchard, LA Cavieres \& CB Anderson. 2009. Análisis cuantitativo de la investigación en invasiones biológicas en Chile: tendencias y desafíos. Revista Chilena de Historia Natural 82: 497-505.

Radashevsky VI \& C Olivares. 2005. Polydora uncinata (Polychaeta: Spionidae) in Chile: an accidental transportation across the Pacific. Biological Invasions 7: 489-496. 
Ramírez ME. 2001. Museos, colecciones y el desafío de la biodiversidad. En: Alveal K \& A Antezana (eds). Sustentabilidad de la biodiversidad, pp. 719-729. Editorial Universidad de Concepción, Concepción.

Ramírez ME, R García-Huidobro \& F Goecke. 2007. Extensión del límite sur de distribución de Asparagopsis armata Harvey (Bonnemaisoniales, Rhodophyta) en la costa de Chile continental, una especie invasora en el Mediterráneo. Noticiario Mensual del Museo Nacional de Historia Natural (Chile) 359: 23-29.

Riascos JM, N Guzmán, J Laudien, ME Oliva, O Heilmayer \& L Ortlieb. 2009. Long-term parasitic association between the boring polychaete Polydora bioccipitalis and Mesodesma donacium. Diseases of Aquatic Organisms 85(3): 209-215.

Rozbaczylo N, F Avilés, M Herve \& M Godoy. 2007. First report of Dodecaceria sp. (Polychaeta: Cirratulidae), in red abalone in Chile. Journal of Shellfish Research 26(3): 855857.

Ruiz GM, PW Fofonoff, JT Carlton, MJ Wonham \& AH Hines. 2000. Invasion of coastal marine communities in North America: Apparent patterns, processes, and biases. Annual Review of Ecology and Systematics 31: 481-531.

Soto D, F Jara \& CA Moreno. 2001. Escaped salmon in the inner seas, southern Chile: Facing ecological and social conflicts. Ecological Applications 11: 1750-1762.

Stachowicz JJ \& JE Byrnes. 2006. Species diversity, invasion success, and ecosystem functioning: disentangling the influence of resource competition, facilitation, and extrinsic factors. Marine Ecology Progress Series 311: 251-262.

Stotz WB, L Caillaux \& J Aburto. 2006. Interactions between the Japanese abalone Haliotis discus hannai (Ino 1953) and Chilean species: consumption, competition, and predation. Aquaculture 255(1): 447-455.

Tarifeño E, R Galleguillos, A Llanos-Rivera, D Arriagada, S Ferrada, CB Canales-Aguirre \& M Seguel. 2012. Erroneous identification of the mussel, Mytilus galloprovincialis (Lamarck 1819) as the specie, Mytilus chilensis (Hupe 1854) in the Bay of Concepcion, Chile. Gayana 76(2): 167-172.
Toro JE, JA Ojeda, AM Vergara, CG Castro \& AC Alcapan. 2005. Molecular characterization of the Chilean blue mussel (Mytilus chilensis Hupe 1854) demonstrates evidence for the occurrence of Mytilus galloprovincialis in southern Chile. Journal of Shellfish Research 24(4): 1117-1121.

Turon X, JI Cañete, J Sellanes, RM Rocha \& S LópezLegentil. 2016. Too cold for invasions? Contrasting patterns of native and introduced ascidians in subantarctic and temperate Chile. Management of Biological Invasions 7(1): 77-86.

Vargas L, P Quijón \& C Bertrán. 2005. Polychaete infestation in cultured abalone (Haliotis rufescens Swainson) in southern Chile. Aquaculture Research 36: 721-724.

Villaseñor-Parada C \& PE Neill. 2011. Distribución espacial de epifitos en el talo de la macroalga introducida Codium fragile subsp. tomentosoides en el submareal de Caldera. Revista de Biología Marina y Oceanografía 46(2): 257-262.

Villaseñor-Parada C, EC Macaya, LM Jara \& PE Neill. 2013. Variación temporal y espacial en la producción de gametangios de la macroalga exótica Codium fragile subsp. tomentosoides (Suringar) Hariot (Chlorophyta: Bryopsidales) en el submareal de Caldera. Revista de Biología Marina y Oceanografía 48(1): 213-218.

Villaseñor-Parada C, A Pauchard \& EC Macaya. 2014. Expansión del rango de distribución de la especie introducida Schottera nicaeensis (Rhodophyta: Gigartinales) en la costa chilena: ¿evidencia de una invasión? Boletín de la Red Latinoamericana para el Estudio de Especies Invasoras 4: 19-27.

Westfall K \& JA Gardner. 2013. Interlineage Mytilus galloprovincialis Lmk. 1819 hybridization yields inconsistent genetic outcomes in the Southern hemisphere. Biological Invasions 15(7): 1493-1506.

Zapata M, F Silva, Y Luza, M Wilkens \& C Riquelme. 2007. The inhibitory effect of biofilms produced by wild bacterial isolates to the larval settlement of the fouling ascidia Ciona intestinalis and Pyura praeputialis. Electronic Journal of Biotechnology 10(1): 149-159. 
Suplemento. Listado de los 71 trabajos utilizadosen el análisis de la literatura relacionada con Ecología de Invasiones Marinas en Chile /

List of the 71 papers used in the analysis of the literature related to Marine Invasion Ecology in Chile

1. Alvarado JL, R Pinto, P Marquet, C Pacheco, R Guiñez \& JC Castilla. 2001. Patch recolonization by the tunicate Pyura praeputialis in the rocky intertidal of the Bay of Antofagasta, Chile: evidence for self-facilitation mechanisms. Marine Ecology Progress Series 224: 93-101.

2. Astorga M, R Guiñez, JC Ortiz \& JC Castilla. 2002. Phenotypic and genetic variation in tunicate Pyura praeputialis (Heller, 1878) in the northern sac of the Antofagasta Bay. Revista Chilena de Historia Natural 75: 515-526.

3. Astudillo JC, M Bravo, CP Dumont \& M Thiel. 2009. Detached aquaculture buoys in the SE Pacific: a potential dispersal vehicle for associated organisms. Aquatic Biology 5:219-231.

4. Avilés F, N Rozbaczylo, M Herve \& M Godoy. 2007a. First report of polychaetes from the genus Oriopsis (Polychaeta: Sabellidae) associated with the Japanese abalone Haliotis discus hannai and other native molluscs in Chile. Journal of Shellfish Research 26(3): 863-867.

5. Avilés F, N Rozbaczylo, M Godoy \& G Muñoz. 2007b. The first report of Phoronis sp. (Phoronida) in red abalone (Haliotis rufescens) in Chile. Journal of Shellfish Research 26: 859-861.

6. Borsa P, V Rolland \& C Daguin-Thiébaut. 2012. Genetics and taxonomy of Chilean smooth-shelled mussels, Mytilus spp. (Bivalvia: Mytilidae). Comptes Rendus Biologies 335: 51-61.

7. Camus C, AP Meynard, S Faugeron, K Kogame \& JA Correa. 2005. Differential life history phase expression in two coexisting species of Scytosiphon (Phaeophyceae) in northern Chile. Journal of Phycology 41(5): 931-941.

8. Camus PA. 2005. Introducción de especies en ambientes marinos chilenos: no solo exóticas, no siempre evidentes. Revista Chilena de Historia Natural 78: 155-159.

9. Caro AU, R Guiñez, V Ortiz \& JC Castilla. 2011. Competition between a native mussel and a non-indigenous invader for primary space on intertidal rocky shores in Chile. Marine Ecology Progress Series 428: 177-185.

10. Carrasco MF \& PJ Baron. 2010. Analysis of the potential geographic range of the Pacific oyster Crassostrea gigas (Thunberg, 1793) based on surface seawater temperature satellite data and climate charts: the coast of South America as a study case. Biological Invasions 12(8): 2597-2607.

11. Castilla JC \& R Guiñez. 2000. Disjoint geographical distribution of intertidial and nearshore benthic invertebrates in the Southern Hemisphere. Revista Chilena de Historia Natural 73:585-603.
12. Castilla JC, AG Collins, CP Meyer, R Guiñez \& DR Lindberg. 2002. Recent introduction of the dominant tunicate, Pyura praeputialis (Urochordata, Pyuridae) to Antofagasta, Chile. Molecular Ecology 11: 1579-1584.

13. Castilla JC, $\mathbf{N}$ Lagos \& M Cerda. 2004a. Marine ecosystem engineering by the alien ascidian Pyura praeputialis on a mid-intertidal rocky shore. Marine Ecology Progress Series 268: 119-130.

14. Castilla JC, R Guiñez, AU Caro \& V Ortiz. 2004b. Invasion of a rocky intertidal shore by the tunicate Pyura praeputialis in the Bay of Antofagasta, Chile. Proceedings of the National Academy of Sciences of the United States of America 101(23): 8517-8524.

15. Castilla JC, M Uribe, $\mathbf{N}$ Bahamonde, M Clarke, $\mathbf{R}$ Desqueyroux-Faúndez, H Moyano, N Rozbaczylo, B Santelices, C Valdovinos \& P Zavala. 2005. Down under the southeastern Pacific: marine non-indigenous species in Chile. Biological Invasions 7(2): 213-232.

16. Castilla JC, PH Manríquez, AP Delgado, L Gargallo, A Leiva \& D Radic. 2007. Bio-foam enhances larval retention in a free-spawning marine tunicate. Proceedings of the National Academy of Sciences of the United States of America 104: 18120-18122.

17. Castilla JC, PH Manríquez, AP Delgado, V Ortiz, ME Jara \& M Varas. 2014. Rocky intertidal zonation pattern in Antofagasta, Chile/: invasive species and shellfish gathering. PLoS One 9: 1-10.

18. Cerda M \& JC Castilla. 2001. Diversity and biomass of macroinvertebrates in intertidal matrices of the tunicate Pyura praeputialis (Heller, 1878) in the Bay of Antofagasta, Chile. Revista Chilena de Historia Natural 74: 841-853.

19. Cifuentes M, I Krueger, CP Dumont, M Lenz \& M Thiel. 2010. Does primary colonization or community structure determine the succession of fouling communities? Journal of Experimental Marine Biology and Ecology 395(1): $10-20$.

20. Clarke M \& JC Castilla. 2000. Dos nuevos registros de ascidias (Tunicata: Ascidiacea) para la costa continental de Chile. Revista Chilena de Historia Natural 73(3): 503-510.

21. Clarke M, V Ortiz \& JC Castilla. 1999. Does early development of the Chilean tunicate Pyura praeputialis (Heller, 1878) explain the restricted distribution of the species? Bulletin of Marine Science 65: 745-754.

22. Collantes G \& P Muñoz-Muga. 2009. Massive proliferation of Grateloupia intestinalis (Hooker fil. et Harvey) Setchell ex Parkinson (Rhodophyta, Halymeniaceae), a non-native species in Valparaíso Bay, central Chile. Revista de Biología Marina y Oceanografía 44(2): 527-532. 
23. Consuegra S, N Phillips, G Gajardo \& CG De Leaniz. 2011. Winning the invasion roulette: Escapes from fish farms increase admixture and facilitate establishment of non-native rainbow trout. Evolutionary Applications 4: 660-671.

24. Dumont CP, JD Urriago, A Abarca, CF Gaymer \& M Thiel. 2009. The native rock shrimp Rhynchocinetes typus as a biological control of fouling in suspended scallop cultures. Aquaculture 292: 74-79.

25. Dumont CP, LG Harris \& CF Gaymer. 2011a. Anthropogenic structures as a spatial refuge from predation for the invasive bryozoan Bugula neritina. Marine Ecology Progress Series 427: 95-103.

26. Dumont CP, CF Gaymer \& M Thiel. 2011b. Predation contributes to invasion resistance of benthic communities against the non-indigenous tunicate Ciona intestinalis. Biological Invasions 13(9): 2023-2034

27. Enríquez R \& R Villagrán. 2008. Chile's experience with developing abalone (Haliotis spp.) farming: opportunities and challenges. Revue Scientifique et Technique (International Office of Epizootics) 27(1): 103-112.

28. Flores-Aguilar RA, A Gutierrez, A Ellwanger \& R Searcy-Bernal. 2007. Development and current status of abalone aquaculture in Chile. Journal of Shellfish Research 26(3): 705-711.

29. Gajardo G \& L Laikre. 2003. Chilean aquaculture boom is based on exotic salmon resources: a conservation paradox. Conservation Biology 17: 1173-1174.

30. García de Leaniz C, G Gajardo \& S Consuegra. 2010. From Best to Pest: changing perspectives on the impact of exotic salmonids in the southern hemisphere. Systematics and Biodiversity 8(4): 447-459.

31. Godoy C \& G Jerez. 1998. The introduction of abalone in Chile: ten years later. Journal of Shellfish Research 17(3): 603-605.

32. González A \& B Santelices. 2004. A dichotomus species of Codium (Bryopsydales) is colonizing northern Chile. Revista Chilena de Historia Natural 77: 293-304.

33. González AV, J Beltrán \& B Santelices. 2014. Colonisation and growth strategies in two Codium species (Bryopsidales, Chlorophyta) with different thallus forms. Phycologia 53(4): 353-358.

34. Guiñez R \& JC Castilla. 2001. An allometric tridimensional model of selfthinning for a gregarious tunicate. Ecology 82(8): 2331-2341.

35. Häussermann V \& G Försterra. 2001. A new species of sea anemone from Chile, Anemonia alicemartinae n. sp. (Cnidaria: Anthozoa). An invader or an indicator for environmental change in shallow water? Organisms Diversity Evolution 1:211-224.

36. Häussermann V, MN Dawson \& G Försterra. 2009. First record of the moon jellyfish, Aurelia for Chile. Spixiana 32(1): 3-7.
37. Hilbish TJ, A Mullinax, SI Dolven, A Meyer, RK Koehn \& PD Rawson. 2000. Origin of the antitropical distribution pattern in marine mussels (Mytilus spp.): routes and timing of transequatorial migration. Marine Biology 136: 69-77.

38. Jiménez JE, AM Arriagada, FE Fontúrbel, PA Camus \& MI Ávila-Thieme. 2013. Effects of exotic fish farms on bird communities in lake and marine ecosystems. Naturwissenschaften 100(8): 779-787.

39. Kim MS, EC Yang, A Mansilla \& SM Boo. 2004. Recent introduction of Polysiphonia morrowii (Ceramiales, Rhodophyta) to Punta Arenas, Chile. Botanica Marina 47(5): 389-394.

40. Lopez DA, BA Lopez \& ML Gonzalez. 2008. Shellfish culture in Chile. International Journal of Environment and Pollution 33: 401-431.

41. López DN, PA Arancibia \& PE Neill. 2013. Potential dispersal mechanisms of the cryptogenic anemone, Anemonia alicemartinae. Revista Chilena de Historia Natural 86(3): 369-372.

42. Macaya EC, S Pacheco, A Cáceres \& S Musleh. 2013. Range extension of the non-indigenous alga Mastocarpus sp. along the Southeastern Pacific coast. Revista de Biología Marina y Oceanografía 48(3): 661-665.

43. Madariaga DJ, MM Rivadeneira, F Tala \& M Thiel. 2014. Environmental tolerance of the two invasive species Ciona intestinalis and Codium fragile: their invasion potential along a temperate coast. Biological Invasions 16(12): 2507-2527.

44. Manríquez PH \& JC Castilla. 2010. Fertilization efficiency and gamete viability in the ascidian Pyura praeputialis in Chile. Marine Ecology Progress Series 409: 107-119.

45. Manríquez PH, E Fica, V Ortiz \& JC Castilla. 2014. Bio-incrustantes marinos en el canal de Chacao, Chile: un estudio sobre potenciales interacciones con estructuras manufacturadas por el hombre. Revista de Biología Marina y Oceanografía 49(2): 243-265.

46. Moreno RA, PE Neill \& N Rozbaczylo. 2006. Native and non-indigenous boring polychaetes in Chile: a threat to native and commercial mollusk species. Revista Chilena de Historia Natural 79: 263-278.

47. Neill PE, O Alcalde, S Faugeron, SA Navarrete \& JA Correa. 2006. Invasion of Codium fragile ssp. tomentosoides in northern Chile: a new threat for Gracilaria farming. Aquaculture 259: 202-210.

48. Oróstica MH, RD Otaíza \& PE Neill. 2012. Blades and papillae as likely dispersing propagules in Chilean populations of Mastocarpus sp. (Rhodophyta, Gigartinales). Revista de Biología Marina y Oceanografía 47(1): 109-119.

49. Ortiz M \& W Stotz. 2007. Ecological and eco-social models for the introduction of the abalone Haliotis discus hannai into benthic systems of north-central Chile: sustainability assessment. Aquatic Conservation: Marine and Freshwater Ecosystems 17(1): 89-105. 
50. Oyarzún PA, JE Toro \& JM Navarro. 2013. Comparison of the physiological energetics between Mytilus chilensis, Mytilus galloprovincialis and their hybrids, under laboratory conditions. Aquaculture Research 44: 1805-1814.

51. Oyarzún PA, JE Toro, O Garrido, C Briones \& R Guiñez. 2014. Differences in sperm ultrastructure between Mytilus chilensis and Mytilus galloprovincialis (Bivalvia, Mytilidae): could be used as a taxonomic trait? Latin American Journal of Aquatic Research 42(1): 172-179.

52. Pacheco CJ \& JC Castilla. 2000. Ecología trófica de los ostreros Haematopus palliatus pitanay (Murphy 1925) y Haematopus ater (Vieillot et Oudart 1825) en mantos del tunicado Pyura praeputialis (Heller 1878) en la Bahía de Antofagasta, Chile. Revista Chilena de Historia Natural 73: 533-542.

53. Pacheco CJ \& JC Castilla. 2001. Foraging behavior of the American oystercatcher Haematopus palliatus pitanay (Murphy 1925) on the intertidal ascidian Pyura praeputialis (Heller 1878) in the Bay of Antofagasta, Chile. Journal of Ethology 19(1): 23-26.

54. Provan JIM, S Murphy \& CA Maggs. 2005. Tracking the invasive history of the green alga Codium fragile spp. tomentosoides. Molecular Ecology 14(1): 189-194.

55. Provan J, D Booth, NP Todd, GE Beatty \& CA Maggs. 2008. Tracking biological invasions in space and time: elucidating the invasive history of the green alga Codium fragile using old DNA. Diversity and Distributions 14:343354.

56. Radashevsky VI \& C Olivares. 2005. Polydora uncinata (Polychaeta: Spionidae) in Chile: an accidental transportation across the Pacific. Biological Invasions 7: 489-496.

57. Riascos JM, N Guzmán, J Laudien, ME Oliva, O Heilmayer \& L Ortlieb. 2009. Long-term parasitic association between the boring polychaete Polydora bioccipitalis and Mesodesma donacium. Diseases of Aquatic Organisms 85(3): 209-215.

58. Rozbaczylo N, F Avilés, M Herve \& M Godoy. 2007. First report of Dodecaceria sp. (Polychaeta: Cirratulidae), in red abalone in Chile. Journal of Shellfish Research 26(3): 855-857.

59. Schröder V \& CG De Leaniz. 2011. Discrimination between farmed and free-living invasive salmonids in Chilean Patagonia using stable isotope analysis. Biological Invasions 13(1): 203-213.

60. Sepúlveda M, I Arismendi, D Soto, F Jara \& F Farias. 2013. Escaped farmed salmon and trout in Chile: Incidence, impacts, and the need for an ecosystem view. Aquaculture Environment Interactions 4: 273-283.

61. Soto D, F Jara \& CA Moreno. 2001. Escaped salmon in the inner seas, southern Chile: Facing ecological and social conflicts. Ecological Applications 11: 1750-1762.
62. Stotz WB, L Caillaux \& J Aburto. 2006. Interactions between the Japanese abalone Haliotis discus hannai (Ino 1953) and Chilean species: consumption, competition, and predation. Aquaculture 255(1): 447-455.

63. Tarifeño E, R Galleguillos, A Llanos-Rivera, D Arriagada, S Ferrada, CB Canales-Aguirre \& M Seguel. 2012. Erroneous identification of the mussel, Mytilus galloprovincialis (Lamarck 1819) as the specie, Mytilus chilensis (Hupe 1854) in the Bay of Concepcion, Chile. Gayana 76(2): 167-172.

64. Toro JE, JA Ojeda, AM Vergara, CG Castro \& AC Alcapan. 2005. Molecular characterization of the Chilean blue mussel (Mytilus chilensis Hupe 1854) demonstrates evidence for the occurrence of Mytilus galloprovincialis in southern Chile. Journal of Shellfish Research 24(4): 11171121.

65. Toro JE, PA Oyarzun, C Penaloza, A Alcapan, V Videla, J Tilleria, M Astorga \& V Martinez. 2012. Production and performance of larvae and spat of pure and hybrid species of Mytilus chilensis and M. galloprovincialis from laboratory crosses. Latin American Journal of Aquatic Research 40: 243-247.

66. Vargas L, P Quijón \& C Bertrán. 2005. Polychaete infestation in cultured abalone (Haliotis rufescens Swainson) in southern Chile. Aquaculture Research 36: 721-724.

67. Villaseñor-Parada C \& PE Neill. 2011. Distribución espacial de epifitos en el talo de la macroalga introducida Codium fragile subsp. tomentosoides en el submareal de Caldera. Revista de Biología Marina y Oceanografía 46(2): 257-262.

68. Villaseñor-Parada C, EC Macaya, LM Jara \& PE Neill. 2013. Variación temporal y espacial en la producción de gametangios de la macroalga exótica Codium fragile subsp. tomentosoides (Suringar) Hariot (Chlorophyta: Bryopsidales) en el submareal de Caldera. Revista de Biología Marina y Oceanografía 48(1): 213-218.

69. Westfall KM \& JPA Gardner. 2010. Genetic diversity of Southern hemisphere blue mussels (Bivalvia: Mytilidae) and the identification of non-indigenous taxa. Biological Journal of the Linnean Society 101: 898-909.

70. Westfall KM \& JA Gardner. 2013. Interlineage Mytilus galloprovincialis Lmk. 1819 hybridization yields inconsistent genetic outcomes in the Southern hemisphere. Biological Invasions 15(7): 1493-1506.

71. Zapata M, F Silva, Y Luza, M Wilkens \& C Riquelme. 2007. The inhibitory effect of biofilms produced by wild bacterial isolates to the larval settlement of the fouling ascidia Ciona intestinalis and Pyura praeputialis. Electronic Journal of Biotechnology 10(1): 149-159. 